\title{
Osteoinduction and Antiosteoporotic Performance of Hybrid Biomaterial Chitosan-Bioactive Glass Graft: Effects on Bone Remodeling
}

\author{
Jebahi $\mathbf{S}^{1,2}$, Oudadesse $\mathrm{H}^{1 *}$, Faouzi FZ1, Elleuch $\mathrm{J}^{2}$, Rebai $\mathrm{T}^{3}$, Keskes $\mathrm{H}^{3}$, Mostafa $\mathrm{A}^{1}$, El Feki $\mathrm{A}^{2}$ and El Feki $\mathrm{H}^{4}$ \\ ${ }^{1}$ UMR-CNRS 6226, Campus de Beaulieu, Université de Rennes 1, 263 Avenue du Général Leclerc, 35042 Rennes, France \\ ${ }^{2}$ Animal Ecophysiology Laboratory, Sfax Faculty of Science, Department of Life Sciences, Sfax, Tunisia \\ ${ }^{3}$ Histology, Orthopaedic and Traumatology laboratory Sfax Faculty of Medicine Sfax, Tunisia \\ ${ }^{4}$ Science Materials and Environment laboratory, Sfax Faculty of Science, Sfax, Tunisia
}

\begin{abstract}
Osteoinductive and antiosteroporotic phenomena could be created by using synthetic biomaterials for applications in bone surgery.

In the present study, $\mathrm{CH}$-based bioactive glass (BG-CH) with $17 \mathrm{wt} \%$ chitosan was elaborated by a freeze-drying process. BG-CH was implanted in the muscle and in the femoral condyles of ovariectomised rats. Grafted tissues were carefully removed for physico-chemical and histological analysis.

Several physic-chemical techniques (XRD, FT-IR, MEB, ICP-OES and NMR) were employed to highlight the effects of chitosan on the glass matrix before and after implantation.

The results of the study show that despite the non-additional osteogenic cells or agents, BG-CH is endowed with an osteoinductive property. After 8 weeks, ${ }^{13} \mathrm{C}$ NMR spectra showed the characteristic signals of $\mathrm{\gamma}$-carbons of the hydroxyproline (71 ppm) abundant in collagen.

Y-carboxyglutamate (55 ppm), which occurs in several other bone proteins like osteocalcin, indicating the BG$\mathrm{CH}$ degradation and the dominance of the bone tissue formation. Moreover, this study showed a rise in Ca and $\mathrm{P}$ ion concentrations in the implanted microenvironment, leading to the formation/deposition of Ca-P phases. Trace elements such as $\mathrm{Zn}$ and $\mathrm{Fe}$ were detected in the newly-formed bone and involved in the bone healing. The study highlights the suitability and the extensive applications of BG-CH composites and the clinically useful therapy in regenerative medicine.
\end{abstract}

Keywords: Bioactive glass; Chitosan; Composite; Grafting; Osteoinduction; Anti osteoporosis; MAS-NMR; Bone regeneration

\section{Introduction}

Recent studies demonstrated that bioglass can be considered as a promising way of promoting tissue repair. It shows a superior in vitro apatite-forming ability, and is able to directly bond to living bone in vivo [1]. Although the bioactive glass has osteoconductive ability, it does not possess any osteoinductive property [2,3]. In addition, even though it apparently interesting in terms of bone repairs and regeneration, it does not seem optimal in terms of therapeutic properties. The explosion in tissue engineering research has highlighted the need for new classes of biodegradable polymers. Chitosan $(\mathrm{CH})$ and some of its complexes have been studied for use in a number of biomedical applications. These include wound dressings [4], drug delivery systems [5] and bone graft [6]. It is recognized that some biomaterials do not have an osteoinductive character in the absence of additional osteoinductive agents, such as Bone Morphogenic Proteins (BMPs) [7]. However, these molecules are complicated by a rapid and burst release that provides relatively short-term control [8]. Multiple functional properties such as drug delivery and low immunogenicity have provided ample opportunities for their potential applications. For that, BG-CH with $17 \mathrm{wt} \% \mathrm{CH}$ has been synthesized and studied as a versatile composite for tissue-engineering. Recently, it has been demonstrated that $\mathrm{CH}$ matrix remains porous and acts as a sponge and trapping the growth factors [9]. In our previous study, the specific surface and porosity were determined. BET analysis was performed on the BG-CH biocomposite. The mesoporous material structure was revealed by adsorption isotherm [10]. In addition BG-CH contained a high rate of porosity equal to $81 \%$ which can play an important role of vascularization. In fact, during bone healing, it is well-known that the cells synthesize a number of growth factors. It could act as a putative local regulator of bone remodeling and thus, the idea is that $\mathrm{CH}$ having a microstructure may act as a chelating agent of growth factors and cytokines, which promote the recruitment, proliferation and differentiation of cells. The overall purpose of the BG-CH-based tissue engineering approach is to induce bone formation at extraskeletal sites without the need of additional osteogenic cells or growth factors. In order to assess the bone quality, several biophysical tools have been developed and applied to characterize natural and tissueformed bone. Our pervious study aimed to evaluate the performance of BG-CH implanted in the femoral condyl of an ovariectomised rat by using several physico-chemical techniques [11]. In this work, NMR spectroscopy was used for monitoring the development of the major components of the organic bone matrix, in particular collagen [1214]. In the present study, ${ }^{13} \mathrm{C}$ NMR represents a very useful tool to track the bone organic component. Although previous NMR studies on collagen in hard and soft tissues had to rely on selective isotopic

*Corresponding author: Oudadesse H, UMR-CNRS 6226, Campus de Beaulieu, Université de Rennes 1, 263 Avenue du Général Leclerc, 35042 Rennes, France, E-mail: hassane.oudadesse@univ-rennes1.fr

Received August 07, 2013; Accepted September 25, 2013; Published Septembe 30, 2013

Citation: Jebahi S, Oudadesse H, Faouzi FZ, Elleuch J, Rebai T, et al. (2013) Osteoinduction and Antiosteoporotic Performance of Hybrid Biomaterial ChitosanBioactive Glass Graft: Effects on Bone Remodeling. J Material Sci Eng 2: 128 doi:10.4172/2169-0022.1000128

Copyright: (C) 2013 Jebahi S, et al. This is an open-access article distributed unde the terms of the Creative Commons Attribution License, which permits unrestricted use, distribution, and reproduction in any medium, provided the original author and source are credited. 
labeling [15-18], techniques have now advanced to study the ${ }^{13} \mathrm{C}$ in the native environment with BG-CH bone graft. The aim of this study is to describe and discuss the osteoinduction mechanism and anti osteoporosis phenomenon. Moreover, we aim to analyze the properties of the de novo formed bone after 8 weeks of implantation into the femoral condoyle rat bone using histology, ICP-OES, SEM and NMR spectroscopy.

\section{Materials and Methods}

\section{Biomaterials synthesis}

First, the bioactive glass particles were synthesized via the melting process $[10,19,20]$. To synthesize BG-CH biocomposite, a concentration $(\mathrm{m} / \mathrm{v})$ of chitosan solution $(0.4 \mathrm{~g}$ of $\mathrm{CH}$ mixed in $40 \mathrm{ml}$ of acetic acid) equal to $1 \%$ was used. The $\mathrm{CH}$ polymer with a medium molecular weight was dissolved in $1 \%$ acetic acid aqueous solution (Sigma, France) during $2 \mathrm{~h}$ at room temperature. Mixture of bioactive glass particles and $\mathrm{CH}$ polymer was stirred during $2 \mathrm{~h}$ at room temperature using magnetic stirring at 1,200 rpm (round per minute). After eliminating surplus solution, the mixture was frozen by liquid azotes and placed into a freezedrying during $24 \mathrm{~h}$ to remove the solvent. The obtained composite with dimension size between 40 and $60 \mu \mathrm{m}$ was immersed in $10 \% \mathrm{NaOH}$ solution for two hours and washed several times with deionized water in order to neutralize the residues of acetic acid. Finally, the composite was frozen by liquid azotes and freeze-dried again for $24 \mathrm{~h}$ to completely remove water. The prepared implants were sterilized by $\gamma$-irradiation from a ${ }^{60} \mathrm{Co}$ source gamma irradiation at a dose of $25 \mathrm{~Gy}$ (Equinox, UK) using standard procedures for medical devices.

\section{Animal model}

Female Wistar rats (16 to19 weeks of age), obtained from the central pharmacy, Tunisia, and bred in the central animal house were used in this study. The rats were acclimatized to their new environment for 15 days before the commencement of the study. The animals were fed on a pellet diet (Sicco, Sfax, Tunisia) and water ad libitum. All the animals were kept under climate-controlled conditions $\left(25^{\circ} \mathrm{C} ; 55 \%\right.$ humidity; $12 \mathrm{~h}$ of light alternating with $12 \mathrm{~h}$ of darkness). All rats were randomly divided into five groups. The first group (I): used as negative control (CT). Sixty days after bilateral ovariectomy, Group II, III, IV and $\mathrm{V}$ used respectively as positive control (OVX), implanted with BG (OVX-BG), BG-CH (OVX-BG-CH) and the last one presented empty defects (OVX-NI). The handling of the animals was approved by the Tunisian ethical committee for the care and use of laboratory animals.

\section{Surgical and postoperative protocol}

All surgical interventions were performed under general anaesthesia in aseptic conditions. Anaesthesia was induced with xylazine (7 to 10 $\mathrm{mg} / \mathrm{kg}$ (i.P) ROMPUN ${ }^{\circledR} 2 \%$, Merial; Lyon, France) and ketamine (70 to $100 \mathrm{mg} / \mathrm{kg}$ (i.m) imalgene, Merial; Lyon, France) depending on the body weight. A drilled hole, 3-mm diameter and 4-mm deep, was created on the lateral aspect of the femoral condyle using a refrigerated drill to avoid necrosis. The drill-hole was filled with $10 \mathrm{mg}$ of BG-CH in OVX-BG-CH group and with $10 \mathrm{mg}$ of BG in OVX-BG group. On days 15 and 30 after implant insertion, all rats were sacrificed and specimens were harvested for physicochemical and biological evaluation. To report the osteoinductive capacity, after the skin and fascia had been incised, muscle pouches were carefully made at the dorsal muscle to limit any bleeding. BG and BG-CH samples were implanted in each pouch separately to prevent inter-sample contact.

\section{Preparation of the samples and histological examination}

Implanted muscles and femoral condyles were harvested, fixed in Burdack (formalin) (Sigma, France) and refrigerated. Specimens were dehydrated, using graded solutions of alcohol increasing from 70 to $100 \%$ ETOH (Siphat, Tunisia). The specimens were then infiltrated with methylmethacrylate and embedded (polymerized) before making the inclusion in a mixture of methylmethacrylate (MMA) (PROLABO, France) and glycolmethacrylate (GMA) (PROLABO, France) without prior decalcification. Sections 6 to $7 \mu \mathrm{m}$ thick were debited along a transverse plane using a sliding microtome (Reichert-Jung). Same histology stains are only used to study very specific types of biological tissue.

\section{Physico-chemical analyses}

XRD analyses - structural studies: Obtained compounds, before and after "in vivo" experiments were analyzed by using X-ray Diffraction. Data were collected using a Philips PW3710 diffractometer ( $45 \mathrm{kV}, 40 \mathrm{~mA}$ and $\lambda \mathrm{Cu}-\mathrm{K}=1.540598 \AA$. The crystalline structures of pure glass (BG) and composite glass-chitosan (BG-CH) were highlighted versus time of implantation in femoral condyle.

NMR measurements: The characterization of the organic part of bone mineral was done using ${ }^{13} \mathrm{C}$ solid-state NMR spectroscopy. The measurements were packed in zirconium rotors with a diameter of 2.5 $\mathrm{mm}$, and spun at the magic angle of $54.7^{\circ}$ with a spinning frequency of $15 \mathrm{KHz}$. Powdered samples of about $100 \mathrm{mg}$ were necessary to fill up the rotor of the NMR probe. The deconvolution of the MAS-NMR spectra was performed on the dmfit 2010 software [19].

ICP-OES analyses - kinetic of ossification: Femoral condyl were dried for $24 \mathrm{~h}$ at $65^{\circ} \mathrm{C}$. Dry femurs were weighed accurately and placed in $25-\mathrm{mL}$ tubes. The $2 \mathrm{ml}$ of nitric acid were added. One milliliter of $30 \% \mathrm{H}_{2} \mathrm{O}_{2}$ (Sigma, France) was placed in the tube after $10 \mathrm{~min}$. The volume of the mixture was made up to $500 \mathrm{ml}$ with distilled water. Standard solutions of $\mathrm{Ca}, \mathrm{P}, \mathrm{Na}, \mathrm{Sr}, \mathrm{Si}$ and Fe were used to prepare the working standard solution, and a blank solution. The element concentrations were measured using inductively coupled plasma optical emission spectrometry ICP-OES (Ciros; Spectro Analytical Instrument, Germany).

SEM - study of the morphologies: The Scanning Electron Microscopy (SEM) (Jeol JSM 6301F) was used to identify morphological changes between bone, BG and BG-CH. The collected samples were prefixed with $2.5 \%$ glutaraldehyde solution PBS (phosphate buffer solution, pH 7.4) (Sigma, France) overnight, and then washed with phosphate buffer solution (PBS), $\mathrm{pH}$ 7.4. Then, the samples were post fixed with $2 \%$ osmic acid solution (Sigma, France) (PBS, pH 7.4) for 90 min and dehydrated with alcohol. The samples were then freeze-dried with a freeze-dryer (JFD-300 Electron Optic Laboratory), and were carried out with a vapor deposition system (JFC-1200).

\section{Statistical analysis}

Statistical analysis was performed using Mann-Whitney U-test with SPSS software. $\mathrm{P}<0.05$ was accepted as statistically significant.

\section{Results}

\section{XRD characterization of specimens after the in vivo assays}

After 4 days of BG implantation, X-ray diagram didn't shows any crystallization peak. This halo is highly related to the amorphous structure of BG biomaterial. It didn't exhibit any modification or 
Citation: Jebahi S, Oudadesse H, Faouzi FZ, Elleuch J, Rebai T, et al. (2013) Osteoinduction and Antiosteoporotic Performance of Hybrid Biomaterial Chitosan-Bioactive Glass Graft: Effects on Bone Remodeling. J Material Sci Eng 2: 128. doi:10.4172/2169-0022.1000128

sign of degradation. Four days after surgery, X-ray diagrams of BG$\mathrm{CH}$-bone suggested the presence of 2 peaks with low intensity at $19.5^{\circ}(2 \theta)$ in $(001)$ orientation plane and a new one at $29^{\circ}(2 \theta)$. This new peak highlights the interactions between bioactive glass and the chitosan polymer. An association between the orthorhombic system of chitosan and the amorphous structure of the glass to form a new structure of glass-chitosan composite was suggested [10]. There is a characteristic broad hump at $31.5^{\circ}(2 \theta)$. This broad diffraction pattern is an indication of the predominantly amorphous form of BG-CH. However, 7 days after surgery, peaks at $19.5^{\circ}$ and $29^{\circ}(2 \theta)$ disappeared. This transformation exhibited BG-CH fast resorption and degradation behavior. In this stage, bone tissue can be viewed as a fine layer of organic matrix without deposition of mineral crystals. XRD analysis permits to determine the formation of HAP as an indication of bone consolidation. In fact, the mineral phase of bone consists of small crystals containing calcium and phosphate, called hydroxyapatite [14]. After 15 days of implantation, we noted the presence of new sharp diffraction peaks that are the result of new formation at $25.7^{\circ}$ $(2 \theta)$ in (002) plane orientation. These peaks show that bone healing is emphasized to provide a stable surface on which osteoblasts and/or their precursor cells may migrate and secrete bone matrix. After 30 days of BG-CH exposure, minerals were deposited within collagen fibers in which large amounts of solid mineral crystals were deposited and the registered peaks were more developed. After 60 days, the evaluation of the crystallinity was performed especially by the analysis of (002) and (211) line profile in implanted bone. The implanted bone was presented in the same way as normal bone and the degrees of calcification seemed to be enhanced. XRD analysis indicates that the formation of HAP was more accentuated in BG-CH when compared with BG especially 15 days after surgery (Figure 1).

\section{NMR measurements}

The organic moiety of the bone Extra Cellular Matrix (ECM) was investigated in detail by ${ }^{13} \mathrm{C}$ solid-state MAS NMR. Figure 2 reports the ${ }^{13} \mathrm{C}$ spectrum and the relative assignments of the native bone and BG$\mathrm{CH} /$ bone after implantation. The NMR spectroscopy showed ${ }^{13} \mathrm{C}$ spectra of native femoral condyle bone, where the typical spectral signature of collagen was observed. Most of the signals in the ${ }^{13} \mathrm{C}$ spectra could be assigned by reference to the chemical shifts of the amino acid residues constituting type I collagen. The signal assignments which agreed with literature were attributed. The 182 and $175 \mathrm{ppm}$ signals were consistent with carboxylate/carbonyl carbons which could belong to proteins. The $\gamma$-carbons of the hydroxyproline (71 ppm) were abundant in collagen. $\gamma$-carboxyglutamate $(55 \mathrm{ppm})$, occurs in several other bone proteins like osteocalcin. The $\beta$-carbon of phosphoserine (61 ppm) was found in other bone phosphate carrier proteins. After 4 weeks of BG-CH implantation, ${ }^{13} \mathrm{C}$ spectrum derived essentially from $\mathrm{CH}$ as observed in Figure 2. In fact, at $102.5 \mathrm{ppm}$, the resonance of the carbon $\mathrm{C}(1)$ assigned to the glucosamine unit is observed, while at $95 \mathrm{ppm}$ a much less intense signal due to $\mathrm{C}(1)$ of the acetylglucosamine unit is shown. At $171.3 \mathrm{ppm}$, weak resonances respectively due to the carbonyl C(7) are observable. The NMR spectra obtained after 8 weeks of implantation could be described as a superposition of the NMR spectra of the de novo generated collagen which represents the most ECM abundant organic component. In fact, characteristic signals from all collagen amino acids are obtained with a unique signal at $71.1 \mathrm{ppm}$, which can be assigned to the $\gamma$-carbon of hydroxyproline (Hyp), an amino acid that is not found in other proteins. This was a clear indication that BG-CH degradation had taken place during an implantation time of 8 weeks.

\section{Histological examination}

Muscle implantation and assessment of the osteo-inductive
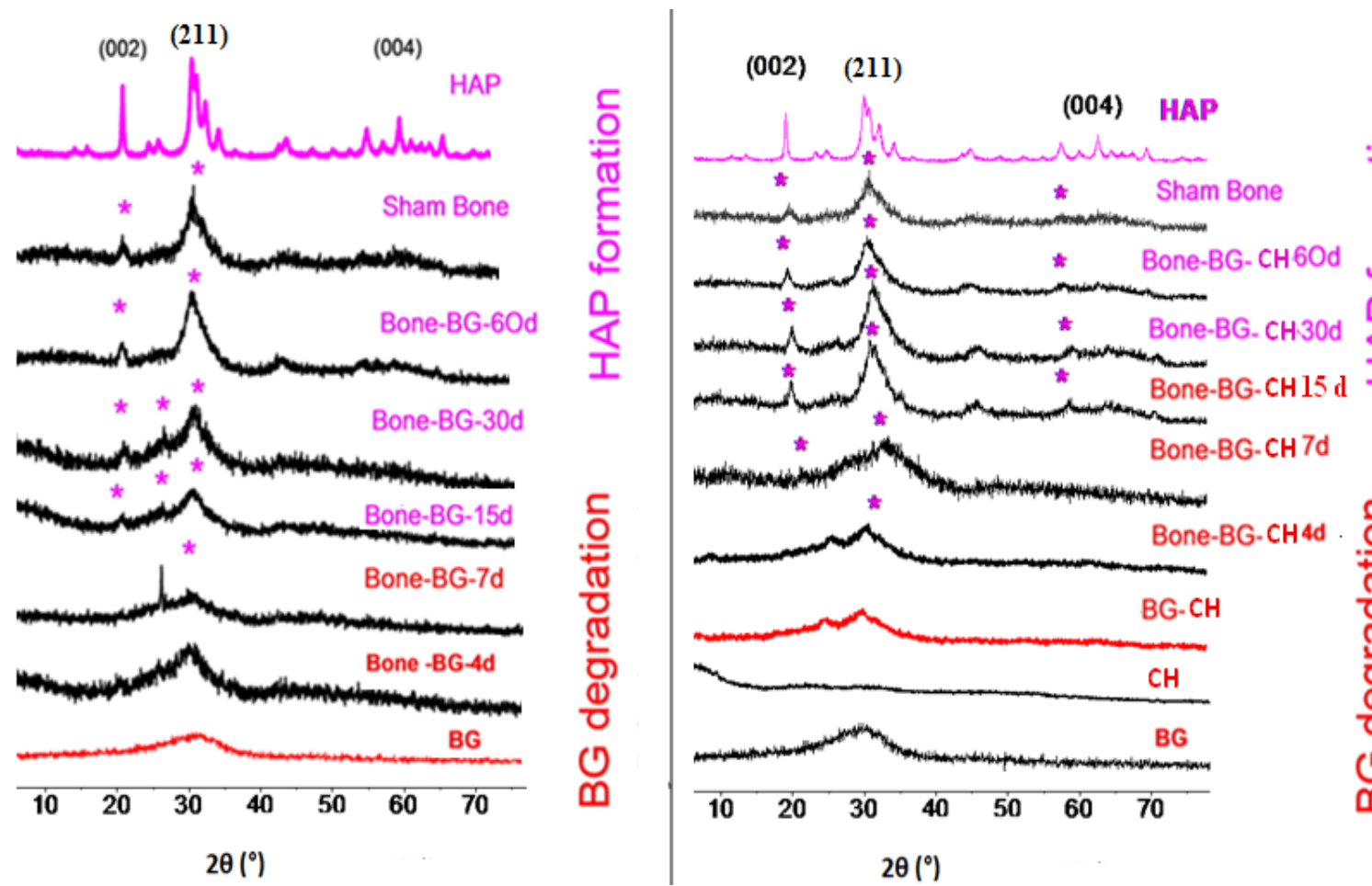

Figure 1: X-ray diffraction from bones implanted by bioglass (BG, in left) and bioglass-chitosan (BG-CH, in right) after 4, 7, 15, 30 and 60 days compared with hydroxyapatite reference spectrum. 


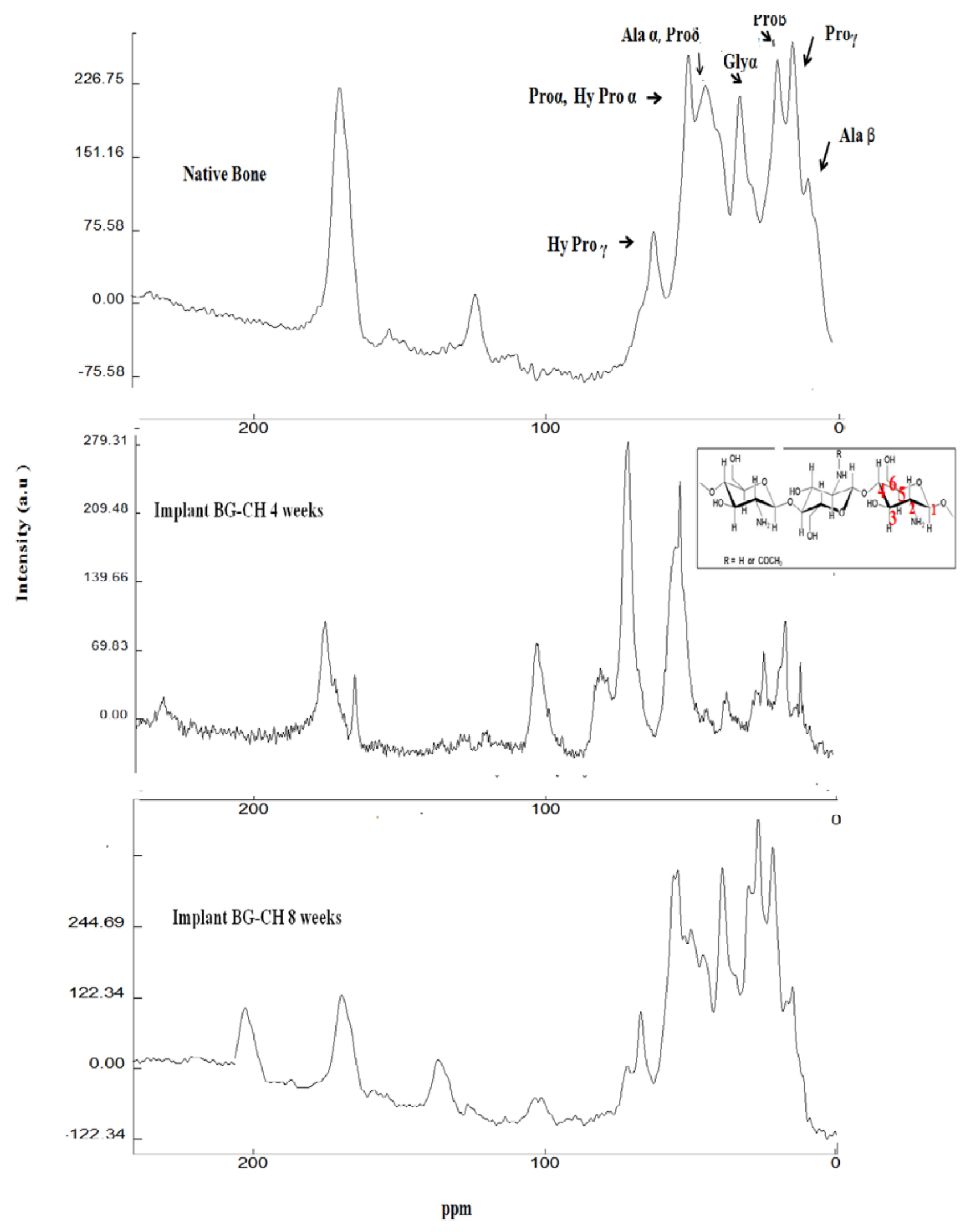

Figure 2: NMR spectra of native rat bone with all detected at a resonance frequency of Ala: Alanine; Arg: Arginine; Glu: Glutamic acid; Gly: Glycine; Hyp: Hydroxyproline; Pro: Proline. Implant BG-CH after 4 weeks and implant BG-CH after 8 weeks. 


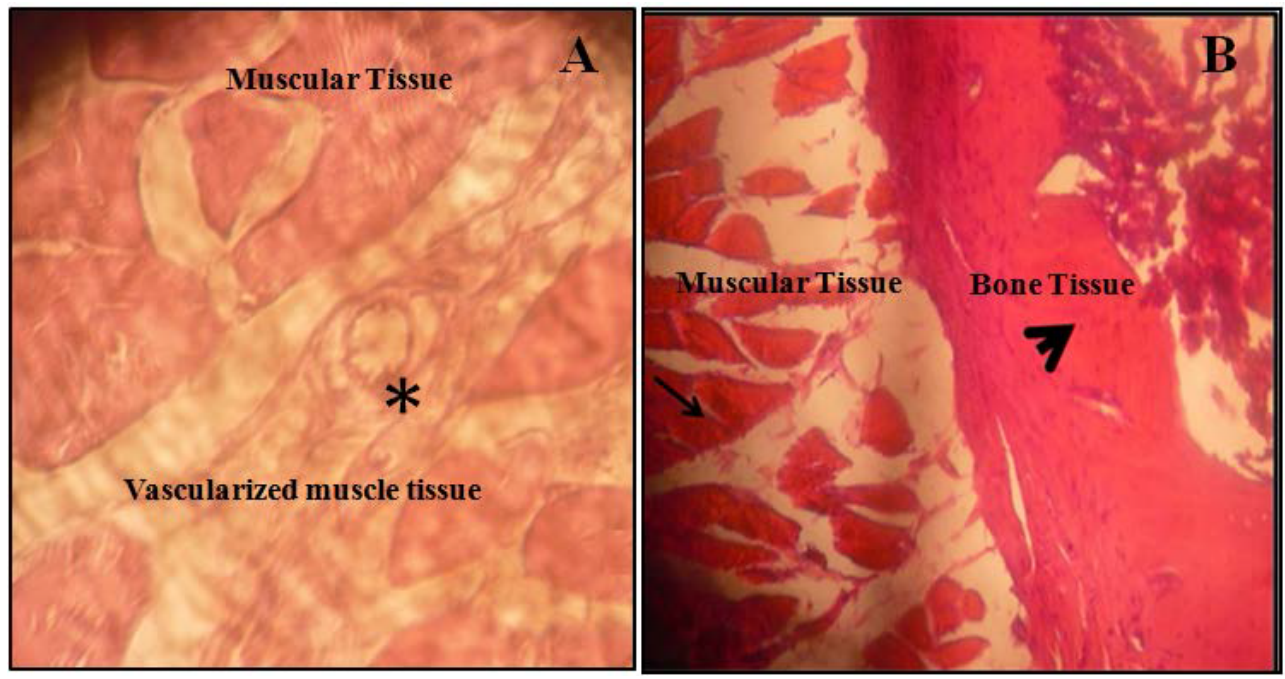

Figure 3: Histological sections of muscular tissue implanted with bioglass (BG). (A) Normal muscular tissue. * indicates blood vessels restoration between fiber muscle in BG treated rat muscles. (B) Bioglass associated with chitosan (BG-CH). Head arrow indicates bone formation. Arrow indicates muscular tissue. Hematoxylin and eosin stain $(A, B,(x 10)$.

ability: Figures 3A and 3B show histological examination of muscular tissues. No bone formation had occurred in all the samples implanted with BG biomaterials after 12 weeks (Figure 3A). However, bone formation was found in the BG-CH implants. This study showed mineralized, newly formed bone that stained well. At the center, newly formed bone islands were connected to each other and the defect was coalesced (Figure 3B). The newly bone tissue was easily distinguished in the contact of the BG-CH surface of the implant. Normal bony structures with osteocytes embedded in the lacunae were observed in the H\&E stained samples. These results highlight the osteoinduction phenomenon in the composite BG-CH unlike that in pure glass BG. ability

Bone implantation and assessment of the anti-osteoporotic

Histological evaluation: After ovariectomy, the trabeculae were disconnected (Figure 4A). After 15 days, a new woven bone was still growing and filling the gap between the host bone and BG implant (Figure 4B). A particular profile of gel layer adhesion to the new bone was observed in the BG-CH implanted group (Figure 4C). Furthermore, histological analysis exhibited the dominance of an osteoid tissue, indicating the characteristic process involved in the formation of new bone in BG implanted group (Figure 4D). However, we noted the dominance of the mineralized tissue in the BG-CH implanted group (Figure 4E). Moreover, the level of the basic structural unit wall width and the trabecular hypertrophy showed a pronounced increase. In fact, BG-CH was shown to limit bone loss inducing a beneficial impact on the trabecular bone microarchitectural properties. It was confirmed by the reduced inter-trabecular space and the higher trabecular thichness when compared to those of BG-treated rats (Figure $4 \mathrm{~F}$ and $4 \mathrm{G}$ ). BG$\mathrm{CH}$ was shown to be able to re-build the cancellous structure and to be replaced with newly formed bone in order to prevent osteoporotic femoral condyl defect (Figure $4 \mathrm{H}$ ). The maturity of the bony matrix was marked by the important number of osteocytes enclosed in a space called the lacuna room (Figure 4J). However, a limited number of osteoclastic cells appeared in the Howship's lacunae-like (Figure 4I).

Scanning Electron Microscopy (SEM): The pore networks were visualized by the scanning electron microscopy (Figure 5). It was observed that $\mathrm{BG}-\mathrm{CH}$ had an average pore size of approximately
$10 \mu \mathrm{m}$ in diameter. The pores could be expected to afford space for bone in growth. In the beginning of the experiment, there was no cell invasion on the $\mathrm{BG}$ or on the $\mathrm{BG}-\mathrm{CH}$ implanted groups (Figure $5 \mathrm{~A}$ and $5 \mathrm{~B})$. After 4 weeks, BG-CH was shown to encourage cell spreading. In fact, the bone surrounding BG-CH presented a highly cellular layer, more advanced ossification and a much larger stimulation of osteoregeneration than those of the BG group. However, only few cells were observed on the surface of BG biomaterial group (Figure $5 \mathrm{C}$ and $5 \mathrm{D}$ ). By the end of the experiment, BG-CH offered a remarkable bone maturation that it could not be readily differentiated from the normal one (Figure 5E). Moreover, Figure 5F can show the BG-CH biodegradation and the mineral crystals disturbance among the bone collagen fibrils.

\section{ICP- OES characterization of newly -formed bone}

As results of ovariectomy, the contents of $\mathrm{Ca}, \mathrm{P}$ and $\mathrm{Na}$ were significantly decreased when compared to those of control group $(\mathrm{P}<0.01)$ as illustrated in Table 1 . As regards, $\mathrm{Sr}$ and $\mathrm{Si}, \mathrm{Na}$ and $\mathrm{Fe}$, no significant differences are observed among control and OVX groups. The implanted group with BG-CH showed that the general reduction of $\mathrm{Si}$ over time concomitant with the change in the release rate of $\mathrm{Ca}$ concentrations. In fact, $\mathrm{Si}$ concentration was decreased from 71 to 50 $\mu \mathrm{g} / \mathrm{g}$ at 15 days and 30 days. However, Ca concentration decreases from 262 to $245 \mathrm{mg} / \mathrm{g}$. These results corroborated the $\mathrm{P}$ content measurements whose investigation shows 139 and $145 \mathrm{mg} / \mathrm{g}$ after 15 and 30 days, respectively. However, $\mathrm{Na}$ content exhibited $9.00 \mathrm{mg} / \mathrm{g}$ in OVX group. These measurements show significant variation after 30 days of post surgery and represent about 10.10 and $12.10 \mathrm{mg} / \mathrm{g}$, respectively in OVX-BG-CH and OVX-BG groups. The concentrations Fe are normalized to the initial bone amount and reveal about $715 \mu \mathrm{g} / \mathrm{g}$. The Sr measurements in OVX bone tissue is of $140 \mu \mathrm{g} / \mathrm{g}$. OVX BG-CH and OVX- BG groups show a significant decrease of Sr, from: 122 and $109 \mu \mathrm{g} / \mathrm{g}$.

\section{Discussion}

\section{Osteinduction property}

The osteoinductive capacity of chitosan-based bioglass (BG-CH) 
Citation: Jebahi S, Oudadesse H, Faouzi FZ, Elleuch J, Rebai T, et al. (2013) Osteoinduction and Antiosteoporotic Performance of Hybrid Biomaterial Chitosan-Bioactive Glass Graft: Effects on Bone Remodeling. J Material Sci Eng 2: 128. doi:10.4172/2169-0022.1000128
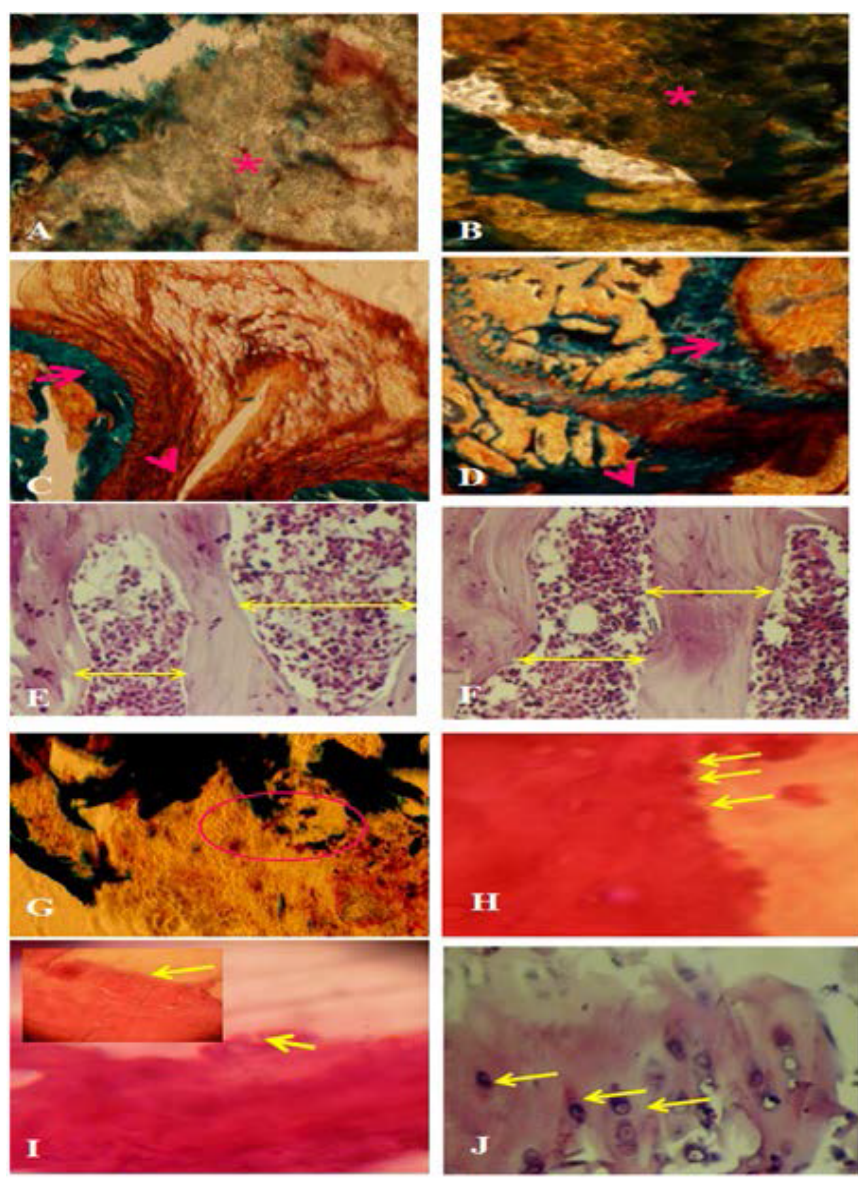

Figure 4: (A) Disconnected trabecular bone in the OVX at 60 days after ovariectomy. (B) A new woven bone in the BG implanted group. (C) A gel layer adhesion to the new bone in the BG-CH implanted group. (D) The osteoid tissue in BG implanted group. (E) The mineralized tissue in the BG$\mathrm{CH}$ implanted group. $(F, G) A$ reduced inter-trabecular space and a higher trabecular thickness. (H) Osteoblast cells. (I) The osteocyte enclosed a space called the lacuna room. (J) Osteoclastic cells in the Howship's lacunae-like.

Goldner's trichrome staining (A, B, C, D, E) (10 x objective). Hematoxylin and eosin stain $(F, G,(x 10) H, I(x 100), J(x 40))$

in Wistar rat has been investigated. BG-CH biomaterials with particle sizes ranging from 40 to $63 \mu \mathrm{m}$ were implanted into the muscles and the bone of the rats for 12 weeks. Our method of characterization by NMR spectroscopy was of great interest to determine the chemical transformation of the newly-formed bone in molecular rearrangement structure, developed in contact with BG-CH composite. To our knowledge, no data is available concerning local response of $\mathrm{BG}-\mathrm{CH}$ as an osteoinductive material, ${ }^{13} \mathrm{C}$ NMR characterization and antiosteoporotic analysis in an ovariectomized rat model.

In the present work, $\mathrm{BG}-\mathrm{CH}$ biomaterials demonstrated the ability to induce bone after 12 weeks in muscular sites without the addition of osteogenic cells or bone growth factors prior to implantation. $\mathrm{CH}$ is considered as a bioactive molecule related to the specific interactions of their surface with the extracellular fluids and cells, ionic exchanges, and cellular activity [21,22]. How the BG-CH microstructure functioned to produce this osteoinductive property was not clear, but some explanations could be found and many hypotheses could be postulated i) Through a dissolution-precipitation process, the development of a calcium phosphate-rich layer might initiate bone formation either by mimicry with the bone mineral structure or by the presence of osteogenic compounds contained naturally in body fluids that might have concentrated at the newly formed mineral layer [23]. When the osteoinduction of calcium phosphate ceramic was reported, the micropore was also reported [24,25], ii) An excellent porosity of bioactive materials signifying more proteins could be absorbed on the surface. The larger surface area could also make easy ion exchanges and bone-like apatite surface formation by dissolution and re-precipitation process [26]. During bone repair, chitosan played an important role in providing cell anchorage site, and structural guidance due to their porosity [10]. They also provided the interface to respond to physiological and biological changes, and to remodel the extracellular matrix to integrate with the surrounding native tissues [27]. Therefore, it was critical to promote cell attachment and migration to regenerate new tissues [28]. To accelerate the repair process, chitosan is implied to incorporate growth factors that are particularly important because it could directly or indirectly promote most cellular processes associated with wound healing, including chemotaxis, stimulation of cell proliferation, and extracellular matrix production [27]. More proteins absorbed on pore surface and the more easily formed apatite layer may facilitate bone formation. The importance of the microstructure in BG-CH -induced osteogenesis does not mean that the microstructure acts as the osteogenic agents in osteoinduction. It may act as a carrier or absorbent of osteogenic agents and then as a release agent [2730]. Thus, BG-CH biomaterial may function as a solid-phase domain for the anchorage of BMP. After BG-CH swelling in the biological
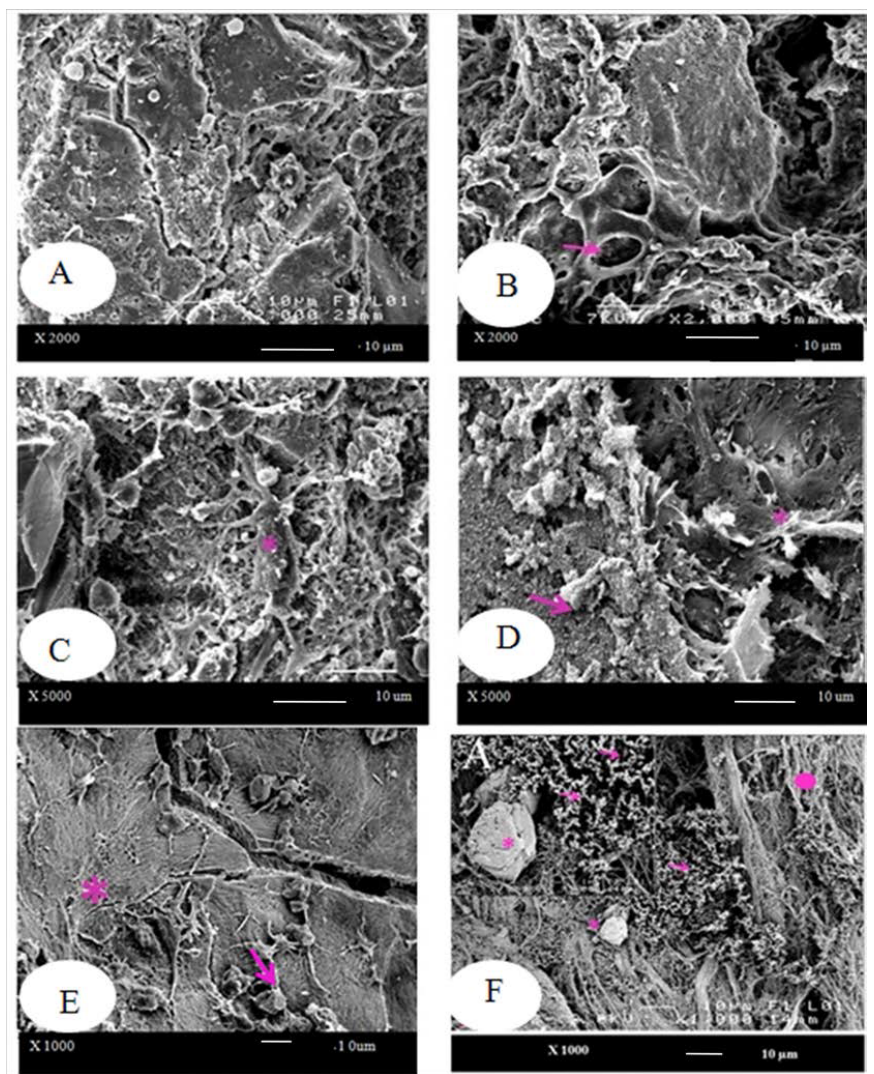

Figure 5: (A, B) Absence of cells invasion on the BG or on the BG-CH implanted groups. (C, D) Highly cellular layer, more advanced ossification and a larger stimulation of osteoregeneration surrounding BG-CH compared with those of the BG group. (E) Degree bone maturation after BG-CH implantation. (F) BG$\mathrm{CH}$ biodegradation and mineral crystals disturbance among the collagen fibrils of bone. 


\begin{tabular}{|c|c|c|c|c|c|c|c|}
\hline & & $\mathrm{Ca}(\mathrm{mg} / \mathrm{g})$ & $P(\mathrm{mg} / \mathrm{g})$ & $S i(\mu g / g)$ & $\operatorname{Sr}(\mu \mathrm{g} / \mathrm{g})$ & $\mathrm{Na}(\mathrm{mg} / \mathrm{g})$ & $\mathrm{Fe}(\mu \mathrm{g} / \mathrm{g})$ \\
\hline $\mathbf{T}$ & & $250 \pm 80$ & $141 \pm 90$ & $25 \pm 09$ & $141 \pm 50$ & $9.4 \pm 90$ & $710 \pm 90$ \\
\hline ovx & & $215 \pm 70^{*}$ & $138 \pm 88$ & $24 \pm 08$ & $140 \pm 90$ & $9.0 \pm 90$ & $710 \pm 90$ \\
\hline \multirow{2}{*}{ BG } & $15 d$ & $260 \pm 68^{*}$ & $139 \pm 70$ & $79 \pm 09^{*}$ & $88 \pm 3^{*}$ & $13.2 \pm 1$ & $740 \pm 2^{*}$ \\
\hline & $30 d$ & $230 \pm 78^{*}$ & $137 \pm 60$ & $62 \pm 04^{*}$ & $109 \pm 2^{*}$ & $12.1 \pm 2$ & $733 \pm 3^{*}$ \\
\hline \multirow{2}{*}{ BG-CH } & $15 d$ & $262 \pm 88^{*}$ & $139 \pm 40$ & $71 \pm 08^{*}$ & $105 \pm 4^{*} ¥$ & $12.12 \pm 5$ & $720 \pm 2 \#^{*}$ \\
\hline & $30 d$ & $245 \pm 78 ¥$ & $145 \pm 50$ & $50 \pm 70^{*} ¥$ & $122 \pm 2^{*} ¥$ & $10.10 \pm 4$ & $715 \pm 3 ¥$ \\
\hline
\end{tabular}

Table 1: Distribution of $\mathrm{Ca}, \mathrm{P}, \mathrm{Si}, \mathrm{Sr}, \mathrm{Na}$, and Fe in newly formed bone of ovariectomised female Wistar rats implanted with bioglass (BG) and chitosan-doped bioglass (BG-CH) for 15 and 30 days. * Significantly different level in the indicated group than control group and $¥$ compared to implanted with bioglass (BG) group.

medium, the gel matrix acts as a sponge through which the fluid accumulates various growth factors ensuring the osteoinductive property. In fact, $\mathrm{CH}$ processes a chain of amino groups along the $\mathrm{CH}$ structure $[31,32]$. Many researchers are now seeking the ability of this amino group to adsorb metal and to chelate ions. This cationic nature is primarily responsible for the electrostatic interactions with anionic glycosaminoglycans (GAG), proteoglycans and other negatively charged molecules. This property is of great interest because a large number of cytokines/growth factors are linked to GAG [33-36]. The BG-CH composite may retain and concentrate growth factors secreted by colonizing cells. From the results presented herein, the addition of the $\mathrm{CH}$ to the biogases materials could be considered as an important factor for triggering osteoinduction.

\section{Anti osteoporotic phenomenon}

BG-CH is endowed with another attractive biological property. In fact, an anti-osteoporotic performace was attributed to $\mathrm{CH}$. In a previous study, this molecule was found to inhibit osteoclastic activity, and is thus widely used to treat patients with osteoporosis [37]. One study demonstrated that incorporation of chitosan to the cement prevents the osteoclastic resorption of the composite biomaterial compared to the cement alone [38]. This inhibitory effect of chitosan on osteoclast resorption activity has important implications on bone formation and bone remodeling after in vivo implantation. Moreover, it is endowed with mitogenic activity toward various types of mesenchymal cells, including osteoblasts [39]. Therefore, it may also enhance bone formation and aid in the differentiation of osteoprogenitor cells [40]. In the present study, during the first 3 days, blood invaded all of the empty spaces between the original bone and the implant. Thereafter, at the end of the first week of implantation, callus and mesenchymal tissues entirely replaced blood while host bone resorption started. Finally, callus, mesenchymal tissues, and host bone have gradually disappeared in favor of newly formed bone while bone remodeling took place [7]. In the current study, $\mathrm{CH}$ induced new bone formation in the distracted zone, and the amount of new bone formation was similar to that in control. These findings suggest that these combined materials are effective in early bony consolidation. They have shown to limit bone loss with a beneficial impact on microarchitectural properties of trabecular bone [41]. In the present study, the level of the basic structural unit wall width and the trabecular hypertrophy showed a pronounced increase. In fact, BG-CH was shown to limit bone loss inducing a beneficial impact on the micro architectural properties. It was confirmed by the reduced inter-trabecular space and the higher trabecular thichness when compared to those of BG-treated rats. The antiosteoprotic property of BG-CH could be explained in part with the different physico-chemical reaction occurring between the bone and the BG-CH surface. After bone implantation, BG-CH biomaterial surfaces sustain dissolution-re precipitation cascades as the result of exchanges at a solid-liquid interface [42]. In terms of surface reactivity, ionic transfers occur from the solid phase to the aqueous liquid via surface hydration of calcium, inorganic phosphate species, present in the biomaterial. Besides, $\mathrm{Ca}$ and $\mathrm{P}$ ions are considered as the major elements responsible for normal bone matrix formation and also for bone mineralization [20]. In this work, after the BG-CH dissolution in the biological system, an equilibrium concentration of $\mathrm{Si}$ was restored when compared to that of the control. The kinetic of the major and trace element is considered as an excellent indication of healthy bone. These results were confirmed by NMR spectroscopy. This technique was of great interest to determine the chemical transformation of the new bone in molecular rearrangement structure, formed in contact with BG-CH composite. It was not superimposed with a BG-CH matrix signal and therefore strongly demonstrated the degradation of this biomaterial. Although peak intensities differed from the native bone samples, characteristic collagen resonances could be easily identified and no major changes of their chemical shifts were observed. This indicated that the de novo formed collagen already had its native structure after 60 days of implantation which was very similar to that of native bone NMR spectrum.

\section{Conclusion}

The results reported herein showed that BG-CH prepared by using a freeze-drying process induced bone formation at non-osseous sites without the need for additional osteogenic cells or agents after 12 weeks of implantation. BG-CH provided support structure for tissue forming cells to synthesize new tissue. The NMR method has the potential for determinnig the quality of the ingrowth of synthetic biomaterials in bone tissue engineering. After 60 days of implantation, the resonance of hydroxyproline $\gamma$-carbons at $71.1 \mathrm{ppm}$ was shown as the most reliable fingerprint of collagen. Our findings suggest that besides the BG-CH osteoinductive property, the antiosteoporotic ability makes of BG-CH a useful material for preventing bone loss associated with postmenopausal osteoporosis.

\section{References}

1. Bhakta S, Faira PE, Salata LA, de Oliveira Neto PJ, Miller CA, et al. (2012) Determination of relative in vivo osteoconductivity of modified potassium fluorrichterite glass-ceramics compared with $45 \mathrm{~S} 5$ bioglass. J Mater Sci Mater Med 23: 2521-2529.

2. Jebahi S, Oudadesse H, El Feki H, Rebai T, Keskes H, et al. (2012) Antioxidative/oxidative effects of strontium-doped bioactive glass as bone graft. In vivo assays in ovariectomised rats. J Appl Biomed 10: 195-209.

3. Jebahi S, Oudadesse H, Jardak N, Khayat I, Keskes H, et al. (2013) Biological therapy of strontium-substituted bioglass for soft tissue wound-healing responses to oxidative stress in ovariectomised rats. Ann Pharm Fr 71: 234242

4. Kirichenko AK, Bolshakov IN, Ali-Riza AE, Vlasov AA (2013) Morphological study of burn wound healing with the use of collagen-chitosan wound dressing Bull Exp Biol Med 154: 692-696.

5. Viota JL, Carazo A, Munoz-Gamez JA, Rudzka K, Gómez-Sotomayor R, et al. (2013) Functionalized magnetic nanoparticles as vehicles for the delivery of the antitumor drug gemcitabine to tumor cells. Physicochemical in vitro evaluation. Mater Sci Eng C Mater Biol Appl 33: 1183-1192.

6. Bojar W, Kucharska M, Ciach T, Koperski L, Jastrzebski Z, et al. (2013) Bone 
Citation: Jebahi S, Oudadesse H, Faouzi FZ, Elleuch J, Rebai T, et al. (2013) Osteoinduction and Antiosteoporotic Performance of Hybrid Biomaterial Chitosan-Bioactive Glass Graft: Effects on Bone Remodeling. J Material Sci Eng 2: 128. doi:10.4172/2169-0022.1000128

Page 8 of 8

regeneration potential of the new chitosan-based alloplastic biomaterial. $\mathrm{J}$ Biomater Appl.

7. Nandi SK, Kundu B, Basu D (2013) Protein growth factors loaded highly porous chitosan scaffold: a comparison of bone healing properties. Mater Sci Eng C Mater Biol Appl 33: 1267-1275.

8. Matsumoto T, Yamada A, Aizawa R, Suzuki D, Tsukasaki M, et al. (2013) BMP-2 Induced Expression of Alx3 That Is a Positive Regulator of Osteoblast Differentiation. PLoS One 8: e68774.

9. Kung S, Devlin H, Fu E, Ho KY, Liang SY, et al. (2011) The osteoinductive effect of chitosan-collagen composites around pure titanium implant surfaces in rats. J Periodontal Res 46: 126-133.

10. Bui XV, Oudadess H, Le Gal Y, Merdrignac-Conanec O, Cathelineau G (2012) Bioactivity behaviour of biodegradable material comprising bioactive glass. Korean J Chem Eng 29: 215-220.

11. Jebahi S, Oudadesse H, Bui XV, Keskes H, Rebai T, et al. (2012) Repair of bone defect using bioglass-chitosan as a pharmaceutical drug: An experimental study in an ovariectomised rat model. Afr J Pharm Pharmaco 6: 1276-1287.

12. Schulz J, Pretzsch M, Khalaf I, Deiwick A, Scheidt HA, et al. (2007) Quantitative monitoring of extracellular matrix production in bone implants by $13 \mathrm{C}$ and $31 \mathrm{P}$ solid-state nuclear magnetic resonance spectroscopy. Calcif Tissue Int 80 : 275-285

13. Xu J, Zhu P, Gan Z, Sahar N, Tecklenburg M, et al. (2010) Natural-abundance 43Ca solid-state NMR spectroscopy of bone. J Am Chem Soc 132: 1150411509.

14. Hu YY, Rawal A, Schmidt-Rohr K (2010) Strongly bound citrate stabilizes the apatite nanocrystals in bone. Proc Natl Acad Sci U S A 107: 22425-22429.

15. Simplaceanu V, Lukin JA, Fang TY, Zou M, Ho NT, et al. (2000) Chain-selective isotopic labeling for NMR studies of large multimeric proteins: application to hemoglobin. Biophys J 79: 1146-1154.

16. Tugarinov V, Kanelis V, Kay LE (2006) Isotope labeling strategies for the study of high-molecular-weight proteins by solution NMR spectroscopy. Nat Protoc 1: $749-754$.

17. Lin MT, Sperling LJ, Frericks Schmidt HL, Tang M, Samoilova RI, et al. (2011) A rapid and robust method for selective isotope labeling of proteins. Methods 55: $370-378$

18. Staunton D, Schlinkert R, Zanetti G, Colebrook SA, Campbell ID (2006) Cellfree expression and selective isotope labelling in protein NMR. Magn Reson Chem 44: S2-S9.

19. Dietrich E, Oudadesse H, Le Floch M, Bureau B, Gloriant T (2009) In vitro Chemical Reactivity of Doped Bioactive Glasses: an Original Approach by Solid-State NMR Spectroscopy. Adv Eng Mater 11: B98-B105.

20. Oudadesse H, Dietrich E, Gal YL, Pellen P, Bureau B, et al. (2011) Apatite forming ability and cytocompatibility of pure and $\mathrm{Zn}$-doped bioactive glasses. Biomed Mater 6: 035006

21. Arunkumar R, Harish Prashanth KV, Baskaran V (2013) Promising interaction between nanoencapsulated lutein with low molecular weight chitosan: characterization and bioavailability of lutein in vitro and in vivo. Food Chem 141: $327-337$.

22. Riyajan SA, Sukhlaaied W (2013) Effect of chitosan content on gel content of epoxized natural rubber grafted with chitosan in latex form. Mater Sci Eng C Mater Biol Appl 33: 1041-1047.

23. Barrère $F$, van Blitterswijk CA, de Groot $K$ (2006) Bone regeneration: molecula and cellular interactions with calcium phosphate ceramics. Int J Nanomedicine 1: 317-332.

24. Yang RN, Ye F, Cheng LJ, Wang JJ, Lu XF, et al. (2011) Osteoinduction by
Ca-P biomaterials implanted into the muscles of mice. J Zhejiang Univ Sci B 12: $582-590$

25. Chan O, Coathup MJ, Nesbitt A, Ho CY, Hing KA, et al. (2012) The effects of microporosity on osteoinduction of calcium phosphate bone graft substitute biomaterials. Acta Biomater 8: 2788-2794.

26. Duan Y, Wu Y, Wang C, Chen J, Zhang X (2003) [A study of bone-like apatite formation on calcium phosphate ceramics in different kinds of animals in vivo] Sheng Wu Yi Xue Gong Cheng Xue Za Zhi 20: 22-25.

27. Inanç B, Eser Elçin A, Koç A, Baloş K, Parlar A, et al. (2007) Encapsulation and osteoinduction of human periodontal ligament fibroblasts in chitosanhydroxyapatite microspheres. J Biomed Mater Res A 82: 917-926.

28. Le Tien C, Lacroix M, Ispas-Szabo P, Mateescu MA (2003) N-acylated chitosan: hydrophobic matrices for controlled drug release. J Control Release 93: 1-13.

29. Muzzarelli RA, Mattioli-Belmonte M, Tietz C, Biagini R, Ferioli G, et al (1994) Stimulatory effect on bone formation exerted by a modified chitosan. Biomaterials 15: 1075-1081.

30. Barbieri D, Yuan H, de Groot F, Walsh WR, de Bruijn JD (2011) Influence of different polymeric gels on the ectopic bone forming ability of an osteoinductive biphasic calcium phosphate ceramic. Acta Biomater 7: 2007-2014.

31. Liao HT, Chen CT, Chen JP (2011) Osteogenic differentiation and ectopic bone formation of canine bone marrow-derived mesenchymal stem cells in injectable thermo-responsive polymer hydrogel. Tissue Eng Part C Methods 17: 11391149.

32. Li N, Bai R (2006) Development of chitosan-based granular adsorbents for enhanced and selective adsorption performance in heavy metal removal. Water Sci Technol 54: 103-113.

33. Yan J, Qi N, Zhang Q (2007) Rabbit articular chondrocytes seeded on collagenchitosan-GAG scaffold for cartilage tissue engineering in vivo. Artif Cells Blood Substit Immobil Biotechnol 35: 333-344.

34. Tan H, Gong Y, Lao L, Mao Z, Gao C (2007) Gelatin/chitosan/hyaluronan ternary complex scaffold containing basic fibroblast growth factor for cartilage tissue engineering. J Mater Sci Mater Med 18: 1961-1968.

35. Tiğli RS, Gümüşderelioğlu M (2009) Chondrogenesis on BMP-6 loaded chitosan scaffolds in stationary and dynamic cultures. Biotechnol Bioeng 104 601-610.

36. Ragetly GR, Slavik GJ, Cunningham BT, Schaeffer DJ, Griffon DJ (2010) Cartilage tissue engineering on fibrous chitosan scaffolds produced by a replica molding technique. J Biomed Mater Res A 93: 46-55

37. Heinemann C, Heinemann S, Bernhardt A, Lode A, Worch H, et al. (2010) In vitro osteoclastogenesis on textile chitosan scaffold. Eur Cell Mater 19: 96-106.

38. Rochet N, Balaguer T, Boukhechba F, Laugier JP, Quincey D, et al. (2009) Differentiation and activity of human preosteoclasts on chitosan enriched calcium phosphate cement. Biomaterials 30: 4260-4267.

39. Jones GL, Motta A, Marshall MJ, El Haj AJ, Cartmell SH (2009) Osteoblast: osteoclast co-cultures on silk fibroin, chitosan and PLLA films. Biomaterials 30: 5376-5384.

40. Hu Y, Cai K, Luo Z, Zhang R, Yang L, et al. (2009) Surface mediated in situ differentiation of mesenchymal stem cells on gene-functionalized titanium films fabricated by layer-by-layer technique. Biomaterials 30: 3626-3635.

41. Wen Z, Zhang L, Chen C, Liu Y, Wu C, et al. (2013) A construction of nove iron-foam-based calcium phosphate/chitosan coating biodegradable scaffold material. Mater Sci Eng C Mater Biol Appl 33: 1022-1031.

42. Oudadesse H, Bui XV, Le Gal Y, Mostafa A, Cathelineau G (2011) Chitosan Effects on Bioactive Glass for Application as Biocopmosite Biomaterial. Int J Biol Biome Eng 5: 49-56 